\title{
Bio Actives from Albizia Lebbeck on Acute Lung Injury/ Acute Respiratory Distress Syndrome Molecular Targets: In-Silico Study
}

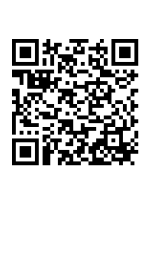

\author{
Priyanka Mishra, Priya Shree, Nikhil Pandey and Yamini Bhushan Tripathi* \\ Department of Medicinal Chemistry, Institute of Medical Sciences, Banaras Hindu University, India
}

Submission: January 26, 2022; Published: February 02, 2022

"Corresponding author: Yamini B Tripathi, Department of Medicinal Chemistry, Institute of Medical Science, India

\begin{abstract}
This contemporary work is prepared with the aim of presenting natural phytoconstituents obtained from Albizia lebbeck (Bronco T) as a remedial option against Acute Lung Injury / Acute Respiratory Distress Syndrome (ARDS). The early onset of non-cardiogenic edema and subsequent gas-exchange impairment caused by a severe inflammatory process known as cytokine storm is acute respiratory distress syndrome (ARDS). The existing strategy includes mechanical ventilation and glucocorticosteroid. However, they pose problems like diaphragm atrophy and down regulated immune response of the host in the lungs of patients with acute respiratory distress syndrome (ARDS), the nuclear regulatory factor NF-kappaB and TLR-4 is activated, which may contribute to increased expression of immune regulatory cytokines and other pro inflammatory mediators including IL-6 and TNF-alpha. Natural products play a pivotal role as it offers minimal and in some cases no toxicity. Albizia lebbeck is known as Sirisha in the folk language and is known for its anti-inflammatory properties. Targeting the above-mentioned receptors with bioactives from Albizia lebbeck would down regulate the signaling pathway and its associated mediators in ARDS. Through insilico study including molecular docking, ADMET and Lipinski analysis, we are trying to narrow down the active phytoconstituent from Albizia lebbeck against ARDS.
\end{abstract}

Keywords: ALI; ARDS; Albizia lebbeck; Autodock-Vina; ADMET; Anti-inflammatory

Abbreviation: ARDS: Acute Respiratory Distress Syndrome; LPS: Lipopolysaccharide; NF- B: Nuclear factor-kappa B; VAP: ventilator-associated pneumonia; AT I: Alveolar type II; AT II: Alveolar type II; HMGB: High mobility group box-1; HIA: Human intestinal absorption

\section{Introduction}

A precipitating cause, such as pneumonia, shock, aspiration of stomach contents, sepsis, or trauma, is invariably present in acute respiratory distress syndrome (ARDS). Because of comorbidities such as sepsis, multiorgan failure, refractory shock, and refractory hypoxemia, patients with ARDS have a significant death rate (50 percent) [1]. Chronic unfavorable outcomes such as fibrosis, tracheal stenosis, pulmonary function decrease, muscle weakness, ambulatory dysfunction, and overall poor quality of life were common among ARDS survivors [2]. Acute inflammation, micro vascular damage, and increased lung vascular and epithelial permeability are all characteristics of ARDS [3]. The immune system is a key player in the etiology of ARDS, according to current knowledge [4]. The severity of lung injury in ARDS patients was linked to serum cytokine and chemokine levels [5]. Infected epithelial cells release cytokines, which attract leukocytes, macrophages, and nearby endothelial cells, causing an increase in cytokine and chemokine production and the symptom known as cytokine storm [6]. Despite significant advances in understanding the etiology of ARDS, little progress has been achieved in developing particular medicines to address the inflammatory damage that occurs in the disease. As a result, medications to treat ARDS, particularly the inflammatory damage associated with the disease, are desperately needed.

Nuclear factor-kappa B (NF-B), a transcription factor, was called after its ability to bind to the enhancer element of the immunoglobulin kappa light-chain of B cells [7]. It is an important inflammatory inducible factor that regulates the transcription of a number of pro inflammatory cytokines, chemokines, and adhesion molecules to mediate the inflammatory response. Punicalagin, for example, inhibits lipopolysaccharide (LPS)induced neuroinflammation, oxidative stress, and memory loss by blocking NF-kB activation [8]. urthermore, when the NF-B signaling pathway is engaged, secreted inflammatory cytokines and chemokines such as IL-1, IL-6, and TNF- $\alpha$ have been shown to have important effects on the course of ALI. Another study confirms that NF-B activation can speed up the transcription of IL-1, IL-6, and TNF- $\alpha$ [5]. TLR-4 plays a role in a variety of inflammatory diseases, including ischemic heart disease and 
ventilator-associated pneumonia (VAP) [9]. Alveolar epithelial cells are divided into two types: alveolar type I (ATI) and alveolar type II (ATI) (ATII). AT1 cells are common in the body and can be readily harmed. When type I cells are damaged, fluid leaks into the alveoli, disrupting regular alveolar clearance. Surfactant secretion is controlled by ATII cells, which is an important role in lowering alveolar tension. In addition, ATII cells have a role in ion transport. Although ATII cells are few in number, they are more resistant to injury $[10,11]$.

A combination of alveolar epithelial cells and capillary vascular cells may be involved in the condition. Endothelial injury, on the other hand, is more common. There is a leakage of fluids and proteins into the interstitium in ARDS due to increased permeability of the capillaries. Fluids, red blood cells, and neutrophils enter the alveolar space through the injured epithelial cells after that. In the exudative phase of ARDS, interstitial and alveolar edema are common [12]. TLR4 is found on both alveolar macrophages and epithelial cells in the lungs. TLR4 detects key ligands like as hyaluronic, LPS, heat shock proteins, and the high mobility group box-1 (HMGB) protein during ARDS propagation [13]. TLR4 activation causes the generation of pro-inflammatory cytokines, which can increase the severity of injuries, as previously stated. Many studies have been conducted in recent years to determine TLR4's exact role in ARDS. The TLR4/nuclear factor (NF)-B pathway could be a key target for inflammatory damage. TLR4 is a pattern recognition receptor from the TLR protein family that activates NF-B and causes the production of inflammatory cytokines and chemokines including TNF- and IL-6 in lung cells. Medicinal plants can be used in this direction as they come with minimum and in some cases no toxicity as well as strengthen immune system via various pathways. Abizia lebbeck, a native tree to Asian and subtropical regions across the world, is a perennial, deciduous tree which is used as a shelter tree for cash crops, for erosion control, as a forage crop and as a source of hardwood [14]. In Ayurveda it is used for various medicinal purposes as it is a non-toxic tree. This tree contains alkaloids, tannins, saponins and flavonoids which have medicinal action, and it is used especially in treating bites and stings from poisonous animals such as snake. Pharmacologically A. lebbeck is used in treatment of various respiratory ailments including bronchial asthma (Table $1 \& 2$ ). In the present study, phytoconstituents of A. lebbeck were analyzed using molecular docking software and the best docked compounds were further processed for druglikeness and ADMET profile analysis using Lipinski Rule of Five and ADMET SAR studies.

Table 1

\begin{tabular}{|c|c|}
\hline Latin & Albizia Lebbeck, Mimosa lebbeck \\
\hline Sanskrit & Siris tree, Shirish \\
\hline English & Woman's tongue, Tibet lebbeck, singer-tree, shack-shack, Lebbeck, Lebbek Tree, Flea Tree, Frywood, Koko \\
\hline Hindi & Shirish, Saras \\
\hline Manipuri & Khok \\
\hline Tamil & Siridam, Vagai \\
\hline Gujrati & Kakiyo,Saras,Sarsado \\
\hline Bengali & Shirish \\
\hline
\end{tabular}

Table 2

\begin{tabular}{|c|c|}
\hline Kingdom & Plantae \\
\hline Order & Fabales \\
\hline Family & Fabaceae \\
\hline Clade & Mimosoideae \\
\hline Genus & Albizia \\
\hline Species & Albizia lebbeck \\
\hline
\end{tabular}

\section{Material and Methods}

\section{Preparation of protein}

RCSB Protein Data Bank (https://www.rcsb.org/) [15] was used to retrieve the crystal structure of TNF-alpha (PDB ID: 2AZ5), TLR4 (PDB ID: 3FXI), NfkB (PDB ID: 1NFK), IL-6(PDB ID: 1ALU). Protein preparation was done with the help of Discovery studio 4.0 by the removal of water molecule and other heteroatoms present in the crystal structure. Further, the active site identification was done for the prepared protein model with the help of Discovery studio 4.0 .

\section{Selection of active phytochemicals-Ligands}

Total 59 active phytochemicals from medicinal plant Albizzia lebbeck were retrieved from literature and database. PubChem compound database (https://pubchem.ncbi.nlm.nih.gov/) was used for retrieval of structure in 2D SDF format. Ligand optimization, energy minimization and conversion of retrieved ligands to 3D PDB formatwere done with the help of Discovery Studio 4.0 .

\section{Molecular docking}

For molecular docking, YASARA software was used [16]. Using YASARA, selected 59 active phytochemicals of Albizzia lebbeck were docked with TNF-alpha (2AZ5), TLR4 (3FXI), NFkb (1NFKB), IL-6(1ALU). For docking study, prepared receptor and ligand files were used to set target and play macro in YASARA 
software. For the calculation of interaction energy between receptor and selected ligands individually, the macro file dockrun_ mcr was used. Afterward, with the help of YASARA software, docked complexes visualize and changed in PDB files for 2D-3D interaction visualization study using Discovery studio 4.0. For the docking calculation study, the result log files from YASARA were taken. Shortening on the basis of binding energy [kcal $/ \mathrm{mol}]$ and dissociation constant [pM], 25 VINA docking runs of the ligand object 2 to the receptor object 1 was done. The compound having more positive binding energies indicates stronger binding, and negative energies indicate no binding.

\section{Drug-likeness and molecular property prediction- ADMET analysis}

The topmost selected active phytochemicals on the basis of binding energy [kcal/mol] and dissociation constant [pM], from Albizzia lebbeck were used for the drug likeness test with the help of Lipinski rule of five (http://www.scfbio-iitd.res.in/software/ drugdesign/lipinski.jsp) [17]. admetSAR server (An Inclusive server for Valuation of Chemical ADMET Properties; (http://lmmd. ecust.edu.cn/admetsar1/predict/) [18] was used for molecular property prediction (ADMET).

\section{Result}

\section{Molecular docking}

Molecular docking study revealed that 19 out of 59 phytochemicals from A. lebbeck showed significant binding affinity with TLR-4, Nf-kB IL-6, TNF-alpha, of inflammatory cascade. Table 3 shows the list of phytochemicals showing significant binding energy ( $\geq 7.0 \mathrm{kcal} / \mathrm{mol}$ ) with above mentioned targets (Figure 1).

Table3: Binding energy (Kcal/mole) of selected phytoconstituents of A. lebbeck against proteins of ARDS. (Phytoconstituents with $>7 \mathrm{Kcal} / \mathrm{mole}$ of binding energy are mentioned here

\begin{tabular}{|c|c|c|c|c|}
\hline Phytoconstituents & TLR-4 & NFKB & IL-6 & TNF-alpha \\
\hline Acacic Acid & 9.02 & 7.12 & 7.86 & 10 \\
\hline Albigenic Acid & 8.42 & 7.79 & 7.4 & 9.88 \\
\hline Albigenin & 9.03 & 8.01 & 7.71 & 10.85 \\
\hline Beta Amyrin & 9.21 & 8.06 & 7.74 & 10.3 \\
\hline Celastrol & 8.5 & 7.9 & 7.51 & 10.44 \\
\hline Friedelin & 8.62 & 7.58 & 7.74 & 10.4 \\
\hline Lupeol & 8.48 & 7.76 & 7.73 & 10.08 \\
\hline Vicenin 2 & 9.64 & 8.15 & 7.6 & 8.52 \\
\hline Alpha Amyrin & 8.63 & 7.73 & 7.59 & 10.46 \\
\hline Triterpenoid & 8.75 & 8.16 & 7.4 & 9.34 \\
\hline Oleanolic Acid & 8.4 & 7.59 & 7.3 & 9.94 \\
\hline Echinocystic Acid & 8.33 & 7.46 & 7.35 & 9.66 \\
\hline Globularicitrin(vitamin P) & 9.94 & 8.02 & 7.19 & 8.52 \\
\hline Tannins & $<7$ & 8.66 & $<7$ & 8.2 \\
\hline Myricitin & 9.31 & 8.01 & $<7$ & 8.6 \\
\hline Reynouritin & 9.34 & 7.95 & $<7$ & 8.19 \\
\hline Melanoxetin & 7.76 & 7 & $<7$ & 8.24 \\
\hline D-catechins & 7.38 & $<7$ & $<7$ & 8.48 \\
\hline Quercetin & 7.68 & 6.84 & 6.58 & 8.55 \\
\hline
\end{tabular}


Figure 1: Bio actives from Albizia lebbeck against molecular target of ALI/ARDS. This is a 2D interaction diagram of ligand-receptor binding interaction where light green bond shows van der waals interaction and dark green bond shows conventional hydrogen bond.

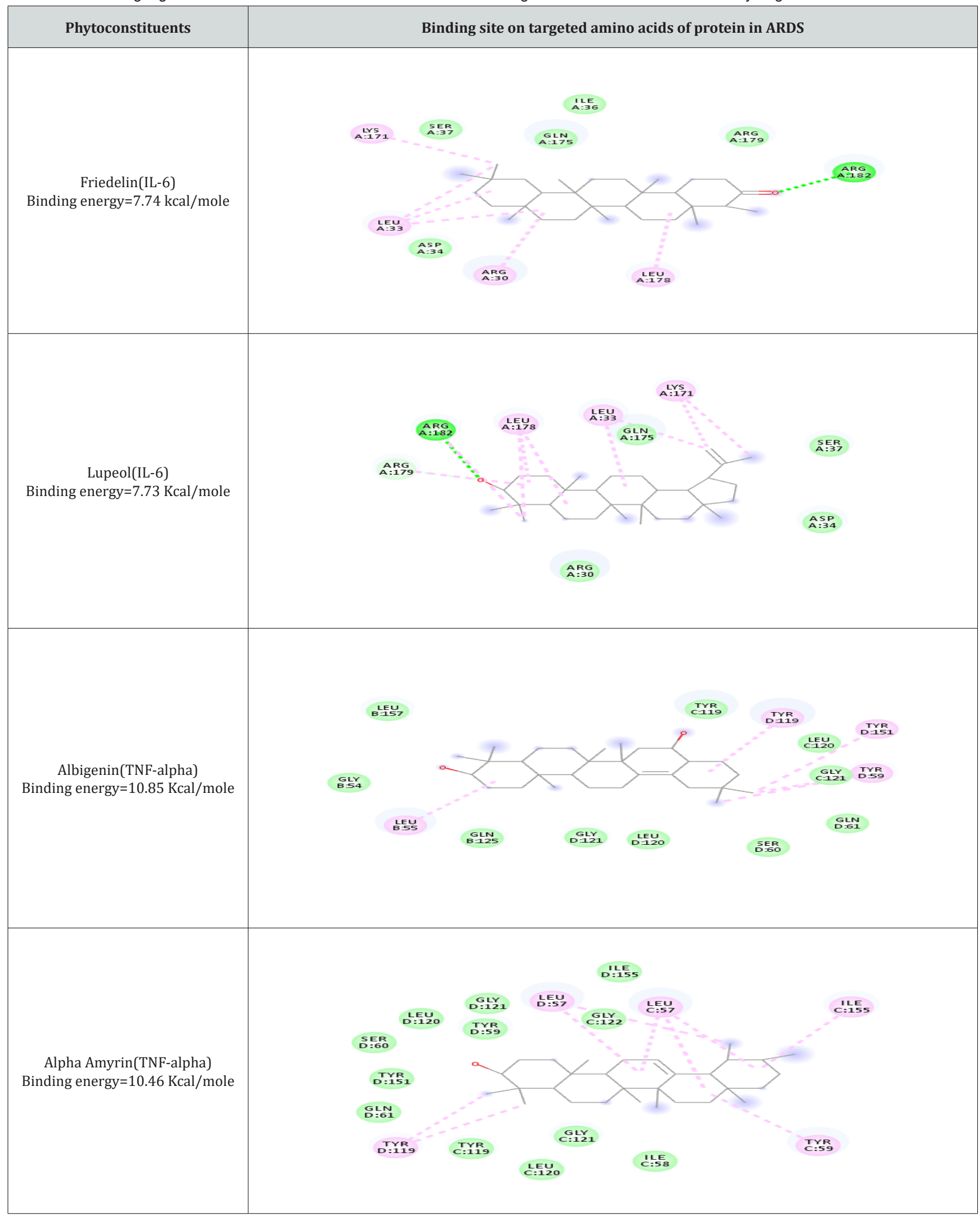




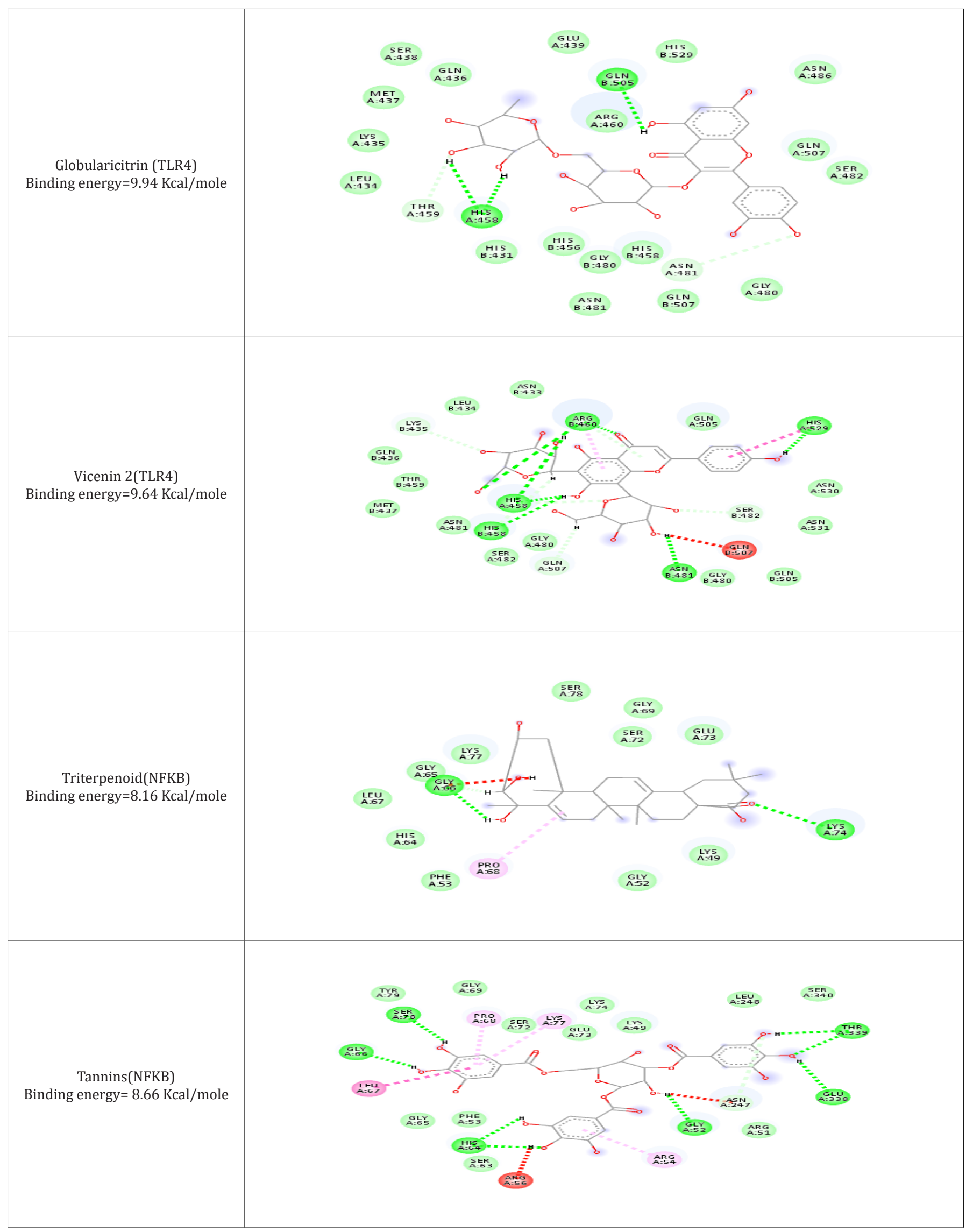




\section{Drug-likeness and ADMET analysis}

Drug-likeness test for best docked compounds was predicted using Lipinski's filter and ADMET molecular property prediction test was performed out by admetSAR server. Lipinski rule of five is a thumb rule of five which helps in differentiating between drug like and non-drug like molecules by obeying its five parameters (Molecular mass, Hydrogen bond donor, Hydrogen bond acceptor,
Log P, and Molar refractivity), it must obey 2 or more of their parameters. Consequently, our best docked compounds (Table 4) follow more than 2 parameters of Lipinski rule of five. admetSAR server provides ADMET profiles of drug candidates. The molecular property profile results indicate positive sign towards human intestinal absorption (HIA) and have no carcinogenic effects, indicating all drugs like properties (Table 4).

Table 4: Drug-likeness and ADMET profile of selected phytocnstituents of A. lebbeck.

\begin{tabular}{|c|c|c|c|c|c|c|c|c|}
\hline Phytoconstituents & $M W<500$ & $\mathrm{HD}<5$ & $H A<10$ & $\log P<5$ & MR (40-130) & HIA & Caco-2 & Carcinogens \\
\hline Albigenin & 426 & 1 & 2 & 7.1019 & 126.56 & 1 & 0.8704 & Non-carcinogenic \\
\hline Friedelin & 426 & 0 & 1 & 8.457 & 129.74 & 1 & 0.8424 & Non-carcinogenic \\
\hline Lupeol & 426 & 1 & 1 & 8.0248 & 130.64 & 0.9974 & 0.8499 & Non-carcinogenic \\
\hline Vicenin 2 & 594 & 11 & 15 & -2.55 & 136.25 & 0.9156 & 0.9096 & Non-carcinogenic \\
\hline Alpha Amyrin & 426 & 1 & 1 & 8.0248 & 130.64 & 1 & 0.831 & Non-carcinogenic \\
\hline Triterpenoid & 472 & 4 & 5 & 4.8492 & 130.89 & 0.9596 & 0.5161 & Non-carcinogenic \\
\hline Globularicitrin & 610 & 10 & 16 & -1.8788 & 137.49 & 0.8041 & 0.9172 & Non-carcinogenic \\
\hline Tannins & 636 & 11 & 18 & -0.2768 & 139.88 & 0.7583 & 0.874 & Non-carcinogenic \\
\hline
\end{tabular}

\section{Discussion}

Since its original description 50 years ago, molecular aetiology and pathophysiology for the development of ALI/ARDS have become better understood. However, "lung-protective ventilation" in mechanically ventilated patients with ARDS is now the best practice, with no specific therapy aimed at lung inflammation. A complex network of proinflammatory signaling pathways and oxidative stress created by a range of cell types in the lungs initiate, amplify, and control the inflammatory response in patients with ARDS. Here in this work we have used in-silico study to screen out the bio actives from the Albizia lebbeck against molecular target of acute lung injury. A. lebbeck is an astringent that is used to cure boils, coughs, eye infections, flu, gingivitis, lung difficulties, chest problems, as a tonic, and to treat abdominal tumors in some cultures [19]. It is a medicinal plant as per Ayurveda the bark can be used to treat inflammations [20]. This formed the hypothesis of the present work as ALI is a clinical condition of respiratory distress involving deregulated inflammatory system. It begins with accumulation of fluid in the alveolar region due to infiltration of neutrophil. Neutrophils serve as the defense mechanism regulated by macrophage polarization [21] in normal condition. However, under the influence of endotoxins the toll like receptors (TLR-4) are activated and they secrete chemokine to flush out the invading pathogens. In ALI/ARDS this mechanism goes out of control especially in cases of septicemia influenced ARDS and creates storm of inflammatory cytokines [22]. From the molecular docking study we found that phytoconstituents from A.lebbeck such as Globularicitrin (9.94 kcal) and Vicenin-2 (9.64 kcal) showed significant binding energy suggesting that they can down regulate TLR- 4 receptors in ARDS condition and can save the patient from deleterious effects. Another pathway involved in pathogenesis of ARDS in Nf-KB. In the lungs of patients with acute respiratory distress syndrome (ARDS), the nuclear regulatory factor NF-kappaB is activated, which may contribute to increased expression of immune-regulatory cytokines and other pro inflammatory mediators [23]. In our study we found that Terpenoids and Tannins have significant binding interaction with Nf-kB suggesting it could control the inflammatory cytokine storm. The major inflammatory cytokines responsible for destructive effect of ARDS are Il-6 and TNF- $\alpha$ [24]. From this in-silico work we found that Friedelin (IL-6), Lupeol (IL-6), Albigenin (TNF-alpha) and Alpha Amyrin (TNF-alpha) were able to inhibit these cytokines by binding with them. All the reported bio actives (Figure 2) from A. lebbeck showed drug-like property as per LPINSKI RULE OF FIVE and were safe as per optimal scoring by ADMETSar software. Despite significant progress in delineating molecular pathways for ALI and ARDS over the previous several decades, these discoveries have not resulted in substantial advances in medical treatment for ARDS patients. From this in-silico work we are reporting for the first time that a medicinal plant from Indian traditional system could be utilized as add on therapy under clinical supervision for management of acute lung injury/acute respiratory distress syndrome. 
Figure 2: Phytoconstituents of Albizia lebbeck with highest binding energy with receptors of ARDS.

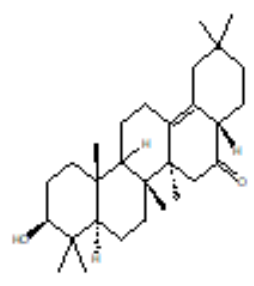

A.BIGENIN

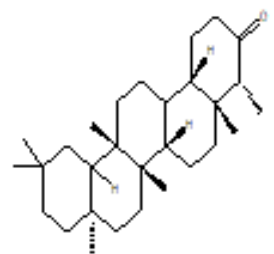

FRIEDFLIN

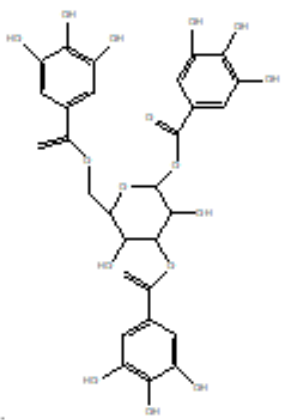

TANINS

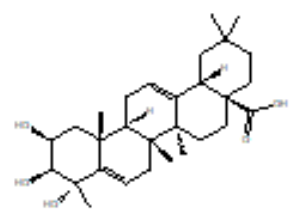

TRITERPENOIDS

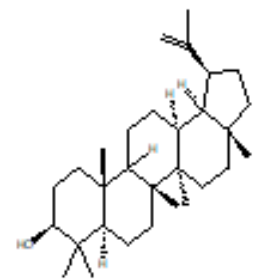

LUPEOL.

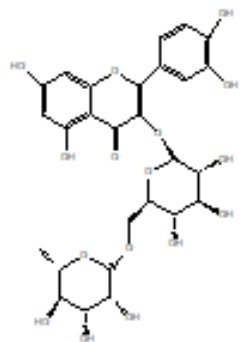

GLOBULARICITRIN

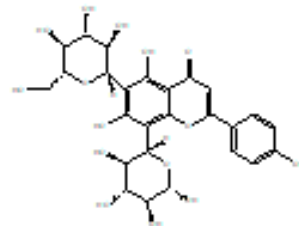

VICENIN-2
8. Verjans E, Kanzler S, Ohl K, Rieg AD, Ruske N, et al. (2018) Initiation of LPS-induced pulmonary dysfunction and its recovery occur independent of T cells. BMC Pulm Med 18(1): 174.

9. Mehrbod P, Ande SR, Alizadeh J, Rahimizadeh S, Shariati A, et al. (2019) The roles of apoptosis, autophagy and unfolded protein response in arbovirus, influenza virus, and HIV infections. Virulence 10(1): 376413.

10. Ganter MT, Roux J, Miyazawa B, Howard M, Frank JA, et al. (2008) Interleukin- $1 \beta$ causes acute lung injury via $\alpha v \beta 5$ and $\alpha v \beta 6$ integrindependent mechanisms. Circ Res 102(7): 804-812.

11. Tomashefski JF (2000) Pulmonary pathology of acute respiratory distress syndrome. Clin Chest Med 21(3): 435-466.

12. Krupa A, Kato H, Matthay MA, Kurdowska AK Proinflammatory AK (2004) Proinflammatory activity of anti-IL-8 autoantibody:IL-8 complexes in alveolar edema fluid from patients with acute lung injury. Am J Physiol Lung Cell Mol Physiol 286(6): L1105-L1113.

13. Tsoyi K, Jang HJ, Lee YS, Kim YM, Kim HJ, et al. (2011) (+)-Nootkatone and (+)-valencene from rhizomes of Cyperus rotundus increase survival rates in septic mice due to heme oxygenase- 1 induction. J Ethnopharmacol 137(3): 1311-1317.

14. Kajaria DK, Gangwar M, Kumar D, Sharma AK, Tilak R, et al. (2012) Evaluation of antimicrobial activity and bronchodialator effect of a polyherbal drug-Shrishadi. Asian Pac J Trop Biomed 2(11): 905-909.

15. Apweiler R, Bairoch A, Wu CH (2004) Protein sequence databases Current Opinion in Chemical Biology 8(1): 76-80.

16. Krieger E, Vriend G, Kelso J (2014) YASARA View-molecular graphics for all devices-from smartphones to workstations. Bioinformatics 
30(20): 2981-2982.

17. Lipinski CA (2004) Lead- and drug-like compounds: The rule-of-five revolution. Drug Discov Today Technol 1(4): 337-341.

18. Cheng F, Li W, Zhou Y, Shen J, Wu Z, et al. (2012) AdmetSAR: A comprehensive source and free tool for assessment of chemical ADMET properties. J Chem Inf Model 52(11): 3099-3105.

19. Babu NP, Pandikumar P, Ignacimuthu S (2009) Anti-inflammatory activity of Albizia lebbeck Benth, an ethnomedicinal plant, in acute and chronic animal models of inflammation. J Ethnopharmacol 125(2): 356-360.

20. Tripathi P, Tripathi YB, Dey PK, S N Tripathi (1983) Tripathi SN. Release of catecholamines from the adrenal medulla by histamine and its prevention by Albezzia lebbek in guinea pigs. Indian J Physiol Pharmacol 27(2): 176-178.
21. Mishra P, Pandey N, Pandey R, Tripathi YB, Todryk S, et al. (2021) Role of Macrophage Polarization in Acute Respiratory Distress Syndrome. J Respir 1(4): 260-272.

22. Deng G, He H, Chen Z, Ou Yang L, Xiao X, et al. (2017) Lianqinjiedu decoction attenuates LPS-induced inflammation and acute lung injury in rats via TLR4/NF-кB pathway. Biomed Pharmacother 96: 148-152.

23. Zhang A, Chi X, Luo G, Hei Z, Xia H, et al. (2013) Mast Cell Stabilization Alleviates Acute Lung Injury after Orthotopic Autologous Liver Transplantation in Rats by Downregulating Inflammation. PLoS One 8(10): e75262.

24. Lentsch AB, Czermak BJ, Jordan JA, Ward PA (1999) Regulation of acute lung inflammatory injury by endogenous IL-13. J Immunol 162(2) 1071-1076. 